\title{
Brain laterality, depression and anxiety disorders: New findings for emotional and verbal dichotic listening in individuals at risk for depression
}

Gerard E. Bruder, Jorge Alvarenga, Karen Abraham, Jamie Skipper, Virginia Warner, Daniel Voyer, Bradley S. Peterson \& Myrna M. Weissman

To cite this article: Gerard E. Bruder, Jorge Alvarenga, Karen Abraham, Jamie Skipper, Virginia Warner, Daniel Voyer, Bradley S. Peterson \& Myrna M. Weissman (2015): Brain laterality, depression and anxiety disorders: New findings for emotional and verbal dichotic listening in individuals at risk for depression, Laterality: Asymmetries of Body, Brain and Cognition, DOI: 10.1080/1357650X.2015.1105247

To link to this article: http://dx.doi.org/10.1080/1357650X.2015.1105247

曲 Published online: 19 Nov 2015.

Submit your article to this journal $\widetilde{ }$

Џ Article views: 19

Q View related articles ¿

View Crossmark data $\nearrow$ 


\title{
Brain laterality, depression and anxiety disorders: New findings for emotional and verbal dichotic listening in individuals at risk for depression
}

\author{
Gerard E. Bruder ${ }^{a, b}$, Jorge Alvarenga ${ }^{b}$, Karen Abraham ${ }^{b}$, Jamie Skipper ${ }^{a}$, \\ Virginia Warner $^{a, b}$, Daniel Voyer ${ }^{c}$, Bradley S. Peterson ${ }^{d, e}$ and Myrna \\ M. Weissman ${ }^{\mathrm{a}, \mathrm{b}}$ \\ ${ }^{a}$ College of Physicians and Surgeons, Columbia University, New York, NY, USA; ${ }^{b}$ New York State \\ Psychiatric Institute, New York, NY, USA; 'Department of Psychology, University of New

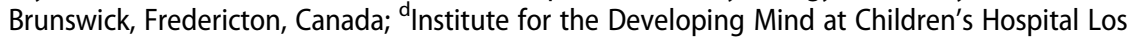 \\ Angeles, Los Angeles, CA, USA; ${ }^{\text {}}$ Department of Psychiatry, University of Southern California, \\ Los Angeles, CA, USA
}

\begin{abstract}
Studies using dichotic listening tests and electroencephalographic (EEG) measures of hemispheric asymmetry have reported evidence of abnormal brain laterality in patients having depressive disorders. We present new findings from a multigenerational study of risk for depression, in which perceptual asymmetry was measured in dichotic listening tests of emotional and verbal processing. Biological offspring and grandchildren of probands with a major depressive disorder (MDD) who were at high risk and those of nondepressed controls who were at low risk were tested on dichotic emotional recognition and consonant-vowel syllable tests. In the emotion test, individuals with a lifetime diagnosis of MDD had a smaller right hemisphere advantage than those without a MDD, but there was no difference between high- and low-risk groups or between those with or without an anxiety disorder. In the syllable test, a smaller left hemisphere advantage was found in individuals with an anxiety disorder compared to those without an anxiety disorder, but there was no difference between highand low-risk groups or between those with or without a MDD. This double dissociation indicates that lifetime diagnosis of MDD and anxiety disorders have a differential impact on lateralized hemispheric processing of emotional and verbal information.
\end{abstract}

ARTICLE HISTORY Received 15 July 2015; Accepted 3 October 2015

KEYWORDS Depression; anxiety disorders; risk for depression; laterality; dichotic listening

Studies have reported abnormal perceptual asymmetry (PA) in patients having a depressive disorder (for a review, see Gadea, Espert, Salvador, \& 
Martí-Bonmatí, 2011). These studies and those using more direct electrophysiological, that is, EEG and event-related brain potential (ERP), measures of hemispheric asymmetry provide evidence of altered brain laterality in depression, which is related to the clinical features of this disorder, including diagnostic subtype and responsiveness to treatments for depression (Bruder, 2003). Individuals at risk for depressive disorder display hemispheric asymmetry for resting EEG measures similar to depressed patients (Bruder et al., 2005; Bruder, Tenke, Warner, \& Weissman, 2007), which suggests that abnormal hemispheric asymmetry may be a trait marker of vulnerability for developing a depressive disorder. We present new dichotic listening findings from a multigenerational study of risk for depression (Weissman et al., 2005), in which measures of PA were obtained in tests using verbal and emotional stimuli for which the left or the right hemisphere is dominant, respectively.

In dichotic listening tests, different stimuli are simultaneously presented to the two ears and accuracy for perceiving stimuli in the right and left ears provides a means for assessing PA. Healthy adults show a right ear advantage for verbal stimuli, representing left hemisphere dominance for language processing, and a left ear advantage for non-verbal or emotional stimuli, representing right hemisphere dominance for processing tonal or prosodic information (Bryden, 1982). Patients with a depressive disorder have been reported to have reduced left ear (right hemisphere) advantage for non-verbal stimuli (for a review, see Bruder, 2003). This was found to be dependent on diagnostic subtype, in that, patients having a melancholic depression with pervasive anhedonia showed no left ear advantage but those having an atypical depression with preserved pleasure capacity had a normal asymmetry (Bruder et al., 1989). In a study measuring brain ERPs during a dichotic complex tone test, healthy controls showed the expected left ear advantage, which was associated with greater amplitude of the $\mathrm{P} 3$ potential over the right hemisphere. Patients having a depressive disorder failed to show either the perceptual or P3 hemispheric asymmetry for tones (Bruder et al., 1995). Findings in depressed patients for verbal dichotic listening tests have been less consistent, with some studies finding abnormally large right ear (left hemisphere) advantage for syllables or words in adolescents and adults having a major depressive disorder (MDD) (Bruder et al., 1989; Pine et al., 2000) and others finding normal asymmetry in depressed patients for perceiving dichotic consonant-vowel syllables (Hugdahl et al., 2003; Moscovitch, Strauss, \& Olds, 1981; Wale \& Carr, 1990).

Differences in dichotic listening findings among studies could be related not only to the diagnostic subtype of depressed patients, but also to comorbid anxiety disorders. Patients having a major depression with a comorbid anxiety disorder had a smaller right ear advantage for a dichotic fusedword test but a larger left ear advantage for complex tones when compared to patients having a major depression without an anxiety disorder (Bruder, 
Wexler, Stewart, Price, \& Quitkin, 1999). Also, patients having social phobia with or without a depressive disorder showed a smaller right ear advantage for dichotic fused-words and syllables than participants without this disorder (Bruder, Schneier, Stewart, McGrath, \& Quitkin, 2004). Electrophysiological studies suggest that comorbidity of anxiety and depression is associated with hyperactivation of right hemisphere regions, which would reduce the PA for dichotic words or syllables in depressed patients (Bruder et al., 1997; Heller, Etienne, \& Miller, 1995). Evidence also suggests that anxious arousal induced by threat of electric shock can eliminate the right ear advantage for dichotic syllables (Asbjörnsen, Hugdahl, \& Bryden, 1992).

Studies using visually presented emotional stimuli have also found evidence of abnormal PA in patients having a depressive disorder (David \& Cutting, 1990; Jaeger, Borod, \& Peselow, 1987; Kucharska-Pietura \& David, 2003; Liotti, Sava, Rizzolatti, \& Caffarra, 1991). Several studies used freevision chimeric faces for which healthy adults have a bias for perceiving faces with a smile to the left hemifield (Levy, Heller, Banich, \& Burton, 1983), which is thought to reflect right hemisphere advantage for processing facial/emotional stimuli. These studies agreed in finding reduced left hemifield bias in patients having a unipolar depressive disorder (David \& Cutting, 1990; Jaeger et al., 1987; Kucharska-Pietura \& David, 2003). Like findings for dichotic listening, this abnormal PA is related to the diagnostic subtype of depression (Bruder et al., 2002). Studies measuring brain ERPs to emotional stimuli have found direct evidence of right parietal hypoactivation in depressed patients (Deldin, Keller, Gergen, \& Miller, 2000; Kayser, Bruder, Tenke, Stewart, \& Quitkin, 2000; Moratti, Rubio, Campo, Keil, \& Ortiz, 2008), which could account for their reduced left hemifield advantage.

In several studies measuring resting EEG, depressed adults and adolescents were found to exhibit abnormal alpha asymmetry indicative of less right than left parietal activity (Bruder et al., 1997; Henriques \& Davidson, 1990; Kentgen et al., 2000; Reid, Duke, \& Allen, 1998), but other studies have not found this parietal asymmetry (Henriques \& Davidson, 1991; Schaffer, Davidson, \& Saron, 1983). These inconsistent findings, as well as those for PAs, may be due to right parietal hyperactivation that accompanies comorbid anxiety, which would counteract the effects of depression (Heller \& Nitschke, 1998; Heller et al., 1995). Heller et al. proposed that the right parietal is involved in anxious arousal and the arousal component of emotion. This possibility is supported by EEG findings in depressed adults and adolescents with a comorbid anxiety disorder who did not show reduced right parietal activity seen for patients having a "pure" depressive disorder (Bruder et al., 1997; Kentgen et al., 2000).

Offspring and grandchildren of depressed patients are at increased risk for developing depressive and anxiety disorders (Warner, Mufson, \& Weissman, 1995; Weissman, Warner, Wickramaratne, Moreau, \& Olfson, 1997; Weissman 
et al., 2005, 2006). Both offspring and grandchildren of probands having a MDD showed greater EEG alpha asymmetry, with less activity over right parietal sites, compared to those at low risk (Bruder et al., 2005, 2007). The alpha asymmetry of high-risk individuals resembles that seen for adults and adolescents having a depressive disorder (Bruder et al., 1997; Henriques \& Davidson, 1990; Kentgen et al., 2000; Reid et al., 1998). Also, EEG evidence of less cortical activity was associated with magnetic resonance imaging (MRI) measures of greater cortical thinning, particularly over right posterior cortex, where differences in alpha were present between high- and low-risk individuals (Bruder et al., 2012). Children at high risk for depression in the mutigenerational study were found to show MRI evidence of cortical thinning across the lateral surface of the right hemisphere, and thinning cortices in right hemisphere was associated with neurocognitive measures of inattention and poorer visual memory (Peterson et al., 2009). Interestingly, one study found that low positive emotionality in children, which has been suggested to confer elevated risk for developing depression, was associated with EEG alpha asymmetry indicating less right than left cortical activity, particularly over parietal sites (Shankman et al., 2005). Thus, EEG, MRI and neurocognitive findings are consistent with the hypothesis that familial risk for depression is accompanied by decreased right hemisphere activity and cortical thickness, impaired emotional processing, and attentional deficits, which increases risk for developing a depressive disorder.

We used dichotic listening tests of emotional and verbal processing to assess hemispheric asymmetries in individuals at high and low risk for depression in the latest wave of our multigenerational study. The pioneering work of Bryden in the study of PA for emotions culminated in the construction of a dichotic listening task that can dissociate verbal and emotional laterality effects (Bryden \& MacRae, 1989). This task has since proved to be fairly reliable both for its verbal (Voyer, 2003) and emotional content (Voyer, Russell, \& McKenna, 2002; Voyer, Bowes, \& Soraggi, 2009). In our study, we focused on the emotional aspect of the task, yielding a left ear advantage in healthy adults (Bryden \& MacRae, 1989; Voyer et al., 2009). This test measures accuracy of emotional recognition for words differing in emotional intonation (angry, sad, happy or neutral) and yields a measure of right hemisphere advantage for prosodic perception. Based on the above EEG and MRI findings, it was predicted that individuals at high risk for depression would differ from those at low risk in showing reduced accuracy and PA in this emotion recognition test. Offspring and grandchildren were also tested in a dichotic consonantvowel syllable task, which not only provides a measure of left hemisphere dominance for verbal processing, but also evaluates the impact of attention on PA (Hugdahl \& Andersson, 1986; Hugdahl et al., 2003). This task uses three different attentional conditions. In a "non-forced" condition, participants are instructed to listen to dichotically presented syllables in both ears and 
report the syllable they heard the clearest, which typically yields a right ear advantage reflecting left hemisphere dominance. In two additional conditions, the participant is asked to attend and report only syllables presented to the right ear (forced-right) or left ear (forced-left). This test assesses accuracy of perceiving dichotically presented syllables under these different attentional demands and yields PA measures for each condition. Given the inconsistency of findings for depressed patients using verbal dichotic listening tests, no prediction was made concerning the PA of high-risk individuals for syllables. However, the finding of cortical thinning of high-risk individuals and the association of cortical thinning with an attention deficit (Peterson et al., 2009) led us to expect a difference in the attentional modulation of performance.

This longitudinal high-risk study also enabled us to examine the impact of lifetime diagnosis of depressive or anxiety disorders on PA in the emotional and verbal dichotic listening tests. Given evidence of reduced right hemisphere advantage for non-verbal dichotic listening in depressed patients (Bruder, 2003), we expected that individuals having a lifetime history of MDD would be particularly likely to show reduced PA in the emotional recognition test. The emotional recognition task that falls within Bryden's legacy allowed us to test this prediction (Bryden \& MacRae, 1989). On the other hand, given findings of reduced left hemisphere advantage for syllables or words in patients having an anxiety disorder (Bruder et al., 1999, 2004), individuals having an anxiety disorder would be most likely to show reduced PA in the dichotic syllable test.

\section{Methods}

\section{Participants}

Full details concerning the selection and assessment of participants in the multigenerational study have been previously described (Warner, Weissman, Mufson, \& Wickramaratne, 1999; Weissman et al., 2005, 2006). Briefly, probands in Generation 1 having a moderate to severe MDD with impairment in functioning were selected from an outpatient clinic in New Haven, CT, for pharmacological treatment. Nondepressed controls were recruited from the same community and had no lifetime history of psychiatric illness based on several interviews. The biological children of probands (second generation) and grandchildren (third generation) of the depressed probands formed the "high-risk" group, and those of the nondepressed controls formed the "lowrisk" group. There were six longitudinal waves of clinical assessments occurring over more than 30 years. The assessment procedures were kept similar across waves. The probands, spouse, offspring and grandchildren were interviewed independently by mental health professionals who demonstrated 
high inter-rater reliability on the interview procedures and who were blind to the clinical status of participants in previous generations. Diagnoses were based on age-appropriate versions of the semi-structured interview, the Schedule for Affective Disorders and Schizophrenia-Lifetime Version (Kaufman et al., 1997; Mannuzza, Fyer, Klein, \& Endicott, 1986; Orvaschel, Puig-Antich, Chambers, Tabriz, \& Johnson, 1982). Diagnoses were based on the best estimate procedure (Leckman, Sholomskas, Thompson, Belanger, \& Weissman, 1982), which involved an independent review of all assessment materials by experienced clinicians. Children aged into the assessments when they were six years old. For children in ages 6-17, both parents and children reported separately on symptoms. The assessment window at each wave covered the time period beginning form the previous completed interview. Thus, although the assessment length for the current period varied, the overall assessment included birth to most recent interview and all resulting diagnoses are lifetime. Current depressive and anxiety symptoms were also assessed on all but seven participants using the Hamilton Rating Scales for Depression (Hamilton, 1967) and Anxiety (Hamilton, 1959) for adults and the Children's Depression Rating Scale (Poznanski, Freeman, \& Mokros, 1985) and Revised Child Manifest Anxiety Scale (Perrin \& Last, 1992) for children 17 years and under. The institutional review board at New York State Psychiatric Institute/Columbia University approved all procedures and informed consent was obtained.

To be eligible for the tests, offspring or grandchildren who were biological descendants of probands had to be older than seven years and have no hearing impairment, history of seizures, head trauma or psychosis. Of 145 eligible participants, one was not tested on the dichotic tests, two were excluded because an audiogram revealed a hearing loss greater than $30 \mathrm{~dB}$ or an ear difference greater than $10 \mathrm{~dB}$, and 14 were not right-handed based on the Edinburgh Handedness inventory (Oldfield, 1971). We contrasted the dichotic listening data for the remaining right-handed offspring (second generation, $n$ $=57$ ) and grandchildren (third generation, $n=71)$ in the high-risk $(n=78)$ and low-risk ( $n=50)$ groups. There was no significant difference in gender or age between the high-risk (41 females, 37 males; mean age $=33.5$ years, $S D=15.7$ ) and low-risk (26 females, 24 males; mean age $=28.1$ years, $S D=13.2$ ) groups. Of the 78 participants in the high-risk group, 43 had a lifetime diagnosis of a MDD and 46 had a lifetime diagnosis of an anxiety disorder. Of the 50 participants in the low-risk group, 12 had a lifetime diagnosis of MDD and 22 had a diagnosis of an anxiety disorder. A comorbid lifetime anxiety disorder was present in 37 of the 55 participants with a MDD, while 31 of the 73 participants without a MDD had an anxiety disorder. An ANOVA including grouping variables of lifetime MDD, Risk and Generation found that participants with a lifetime MDD had greater severity of current depressive symptoms when compared to those without a lifetime MDD, $F(1,113)=4.52, M S E=0.975, p$ 
$=.036, \eta^{2}=.039$ ). Similarly, participants with a lifetime anxiety disorder had greater severity of current anxiety symptoms when compared to those without an anxiety disorder $\left(F(1,113)=3.92, M S E=0.969, p=.050, \eta^{2}\right.$ $=.034$ ). There was, however, no significant difference in current depression or anxiety symptoms between those at high or low risk.

\section{Material and procedures}

An audiogram and dichotic listening tests were obtained during the sixth wave of assessments. Following an audiogram to check for hearing impairment, each participant was tested on the dichotic emotional recognition test, and all but two were then tested on a consonant-vowel test in a sound-attenuated IAC booth. A pre-recorded CD of the emotion recognition test (Voyer et al., 2009) and the consonant-vowel syllable test (Hugdahl \& Andersson, 1986; Hugdahl et al., 2003) were presented using a CD player (SONY CDP-XE400) and stereo receiver (Yamaha RX-395), which allowed adjustment of sound pressure level (SPL) level to both ears for each test. The dichotic stimuli in each test were presented via a matched pair of TDH49 headphones at $73 \mathrm{~dB}$ SPL in each ear.

\section{Audiogram}

Standard procedures were used to measure the hearing levels at 500, 1000 and $2000 \mathrm{~Hz}$ in each ear. ${ }^{1}$

\section{Emotion recognition test}

Details concerning this test and its administration are given in Voyer et al. (2009). The stimuli consisted of four words (power, tower, dower and bower) pronounced in four different emotional tones (angry, happy, sad or neutral). A binaural emotion recognition task was first administered to familiarize participants with recognizing each emotion. In this task, they listened to stimuli (word/emotion combination) and were required to mark the perceived emotion on an answer sheet, which consisted of line drawings of emotional faces representing the four possible emotions (angry, happy, sad or neutral), by crossing out the face corresponding to the emotion they heard clearest from the four alternatives. There were three randomized

\footnotetext{
${ }^{1} \mathrm{After}$ excluding those with a hearing loss greater than $30 \mathrm{~dB}$ or ear difference greater than $10 \mathrm{~dB}$ at $500-$ $2,000 \mathrm{~Hz}$, the hearing levels for participants were well within the normal range (i.e. generally $\leq 20 \mathrm{~dB}$ loss). We cannot rule out the possibility that hearing loss at frequencies higher than $2,000 \mathrm{~Hz}$ could have influenced our findings. It is, however, unlikely that the differences in PA on the emotional recognition test between participants with vs. without a lifetime MDD or differences in PA on the syllable test between participants with vs. without a lifetime anxiety disorder were due to differences in the hearing level. An ANOVA of hearing levels at $500,1,000$ and $2,000 \mathrm{~Hz}$ in each ear indicated that there was no significant difference in the hearing level between these groups and no group by ear interaction (all $p$-values $\geq .28)$. Moreover, neither overall hearing levels or ear difference in hearing levels correlated with PA scores for the emotional recognition test $(r \leq .01, p \geq .67)$ or the syllable test $(r \leq 0.12, p \geq .19)$.
} 
blocks of the 16 different word/emotion combinations resulting for a total of 48 trials with a 5 -s intertrial interval in the binaural task. In the dichotic emotion task, the same stimuli used in the binaural task were presented dichotically with a 5-s intertrial interval. After listening to the stimuli, participants crossed out the faces corresponding to the two dichotically presented emotions (one from each ear). The dichotic task used the 144 possible combinations resulting from the dichotic pairing of the 16 word/emotion stimuli without pairing of the same word or emotion. There were four practice trials and two blocks of 72 test trials, separated by a 40-s rest period. Half of the participants listened to items with the right headphone on the right ear, while the other half of participants had the opposite arrangement in the first 72 item block. Also, to further prevent confounding due to intensity differences between earphones, orientation of the headphones were reversed in the second block of 72 trials.

\section{Consonant-vowel test}

The stimulus materials, test and scoring procedures were provided by Hugdahl and are described in Hugdahl and Andersson (1986) and Hugdahl et al. (2003). The dichotic stimuli consisted of six stop consonants paired with the vowel /a/ to form six consonant-vowel syllables (ba, da, ga, pa, ta and $k a$ ). These syllables were paired with each other in all possible combinations yielding 36 syllable pairs (including 6 homonymic pairs). There were three randomizations of these 36 pairs for each of 3 attentional conditions. Each syllable was approximately $350-400 \mathrm{~ms}$ long, with an intertrial interval of $4 \mathrm{~s}$. Temporal alignment of syllables between right and left channels was set at the energy release in both the consonant and vowel segments of the syllables. There were three conditions with different instructions on how to focus attention. For all conditions, the participant's task was to report the syllable they heard by crossing off one syllable on a response sheet listing the six possible syllables ( $b a, d a, g a, p a, t a$ and $k a$ ). In the non-forced condition, participants were instructed to report the sound they heard, and if they heard two different sounds, they should report the sound they heard most clearly. In the forced-right condition, participants were told to listen only to the right ear and report only the sound they heard in that ear. In the forced-left condition, participants listened only to the left ear and reported only sounds in that ear. The non-forced condition was always presented first, followed by the forced-right and forced-left conditions in a counterbalanced order.

\section{Statistical analyses}

The number of correct responses was computed for right and left ear presentations in each dichotic test. To evaluate our main hypotheses concerning the 
effects of risk for depression and lifetime diagnosis of depression on accuracy and PA in the emotional recognition test, we performed a $2 \times 2 \times 2 \times 2 \times 4$ ANOVA of accuracy scores with between group variables of Risk (high and low), Generation (second and third), and Depression (MDD, NoMDD) as between group variables and Ear (right, left) and Emotion (angry, happy, sad and neutral) as within-subject variables. An ANOVA of accuracy scores in the syllable test was also performed with these same group and ear variables and the variable of condition (non-forced, forced-right and forced-left) to examine the effects of attention. Secondary analyses using the above ANOVA models were conducted to examine the effects of Risk, Generation and lifetime diagnosis of Anxiety Disorder on performance and PA in the dichotic listening tests. Follow-up ANOVA were also conducted to examine group differences in PA for each emotion, and the effects of co-morbidity of MDD and anxiety disorders on PA for emotional recognition and syllable tasks. Spearman's rho correlations examined whether PA (right minus left ear difference) and accuracy scores on each test were related to severity of current depressive and anxiety symptoms. Scores for each adult on the Hamilton Rating Scales for Depression and Anxiety were first converted to standard $Z$-scores and scores for children on the Children's Depression Rating Scale and Revised Child Manifest Anxiety Scale were also converted to Z-scores. Correlations of these depression and anxiety scores (across standard scores for children and adults) with PA and accuracy scores were then computed for each test. Spearman's rho correlations were also calculated between the PA scores for the emotional recognition and syllable tests.

\section{Results}

\section{Emotional recognition test}

\section{Lifetime MDD}

In the first ANOVA, lifetime MDD was used as a grouping variable along with variables of Risk, Generation, Ear and Emotion. As can be seen in Figure 1, the expected PA was found for the emotional recognition test with significantly greater accuracy for the left ear than right ear, $F(1,120)=35.17, M S E=$ $140.83, p<.001, \eta^{2}=.227$. There was also a Depression by Ear interaction, $F$ $(1,120)=8.41, M S E=140.83, p=.006, \eta^{2}=.060$, which was due to the smaller left ear (right hemisphere) advantage in individuals with lifetime MDD compared to those with no $\operatorname{MDD}(t(126)=2.16, p=.041$, Cohen's $d$ $=.38$ ). The difference in accuracy between the right and left ears in those with a lifetime MDD $(M=-3.8, S D=9.7)$ was about half that seen for those with no $M D D(M=-7.0, S D=7.2)$. The smaller left ear advantage in MDD was evident for those at low or high risk for depression and there was no difference in a left ear advantage between high and low-risk groups, which 


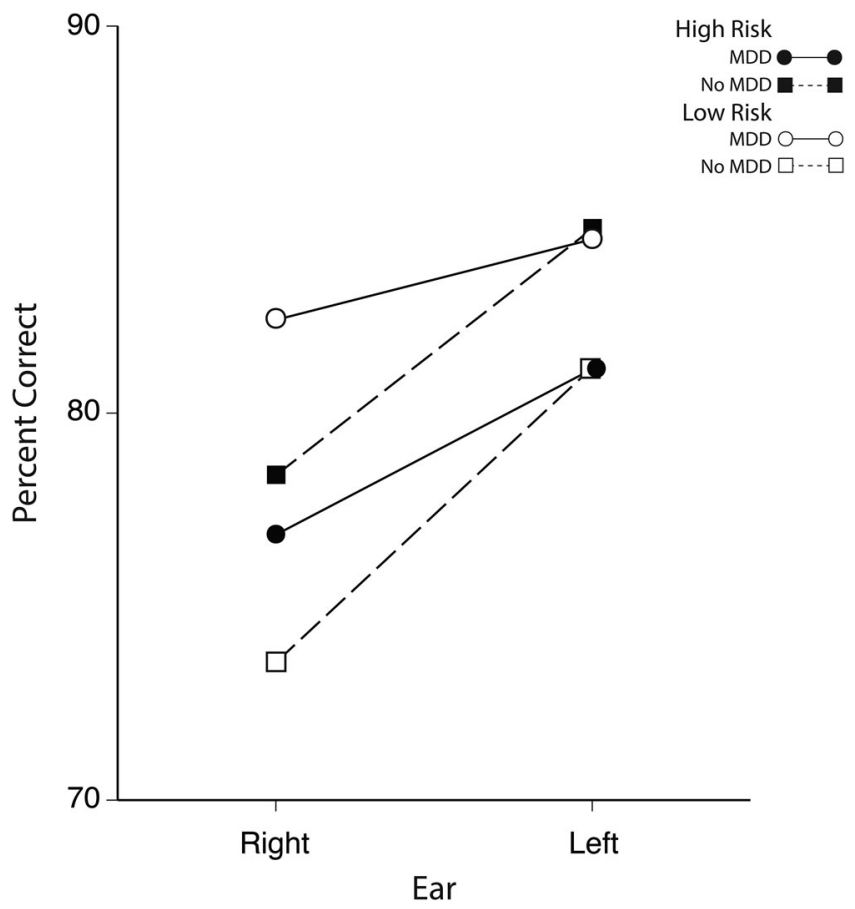

Figure 1. Mean accuracy in emotional recognition test for the right and left ears in highrisk and low-risk groups with or without a MDD.

is indicated by lack of significant interactions of Risk by Ear or Risk by Depression by Ear (all $p$ values $\geq .39$ ). There were also no significant effects of generation, and a separate analysis including sex of participants as a variable did not find significant effects of this variable on the left ear advantage or accuracy levels.

The difference in PA for emotional recognition between individuals with a MDD and those with no MDD was not due to a difference in accuracy levels. There was no significant difference between these groups in either left ear accuracy, $t(126)=0.57, p=.57$ or right ear accuracy, $t(126)=1.16, p=.25$. There was a borderline Risk by MDD interaction, $F(1,120)=3.55, M S E=$ $694.38, p=.062, \eta^{2}=.029$. The basis for this interaction is evident in Figure 1. Among low-risk participants, those with a MDD performed better than those with no MDD, $t(48)=2.26, p=.028$, but among the high-risk participants, there was no significant difference in accuracy between the MDD and no MDD groups, $t(76)=1.13, p=.26$.

There was also a significant Ear by Emotion interaction, $F(3,360)=19.21$, $\left.M S E=62.90, p<.001, \eta^{2}=.138\right)$ and a Depression by Ear by Emotion interaction, $F(3,360)=3.25, p=.02, \eta^{2}=.026$, which are illustrated in Figure 2 . Individuals with no MDD had the largest left ear (right hemisphere) 


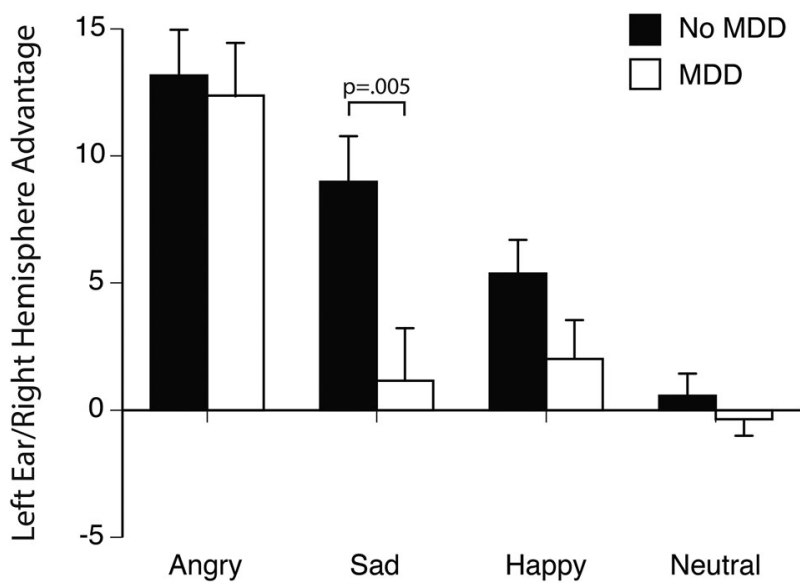

Figure 2. Mean left ear/right hemisphere advantage for angry, sad, happy and neutral items in the emotional recognition test in groups with or without a MDD (error bars $=$ standard errors of the mean).

advantage for angry and sad emotional items (correlated $t$-tests, $t[72]=8.15$ and 5.65, respectively, $p<.001)$, somewhat smaller PA for happy items $(t$ $[72]=4.33, p<.001)$, and no PA for neutral items $(t[72]=0.65, p=.65)$. Individuals with a MDD had a significant left ear advantage only for angry emotional items, $t(54)=5.32, p<.001)$. Those with a MDD had a markedly smaller left ear advantage for sad items compared to those with no MDD $(t[126]=2.85, p=.005$; Cohen's $d=.50)$ and a non-significant trend for a smaller left ear advantage for happy items $(t[126]=1.65, p=.10$; Cohen's $d=.29$ ), but there was no significant group difference in PA for angry or neutral items.

\section{Lifetime anxiety disorder}

When lifetime anxiety disorder was used as a grouping variable in an ANOVA along with variables of Risk, Generation, Ear and Emotion, there was no significant difference in PA between individuals with versus without an anxiety disorder, Anxiety Disorder by Ear interaction: $F(1,120)$ $=2.25, M S E=146.34, p=.14, \eta^{2}=.018$. Those with an anxiety disorder showed the expected left ear advantage (Left ear: $M=82.4, S D=11.4$; Right ear: $M=77.5, S D=11.8$ ) as did those with no anxiety disorder (Left ear: $M=82.5, S D=8.4$; Right ear: $M=76.1, S D=9.2$ ). There was also no significant difference in accuracy levels between those with versus without an anxiety disorder, $F(1,120)=.08, M S E=713.49, p=.78, \eta^{2}=.001$. Moreover, an ANOVA of PA for sad emotional items, in which MDD and anxiety disorder were included as grouping variables, found that the smaller left ear advantage for $M D D$ compared to no $\operatorname{MDD}(F[1,124]=8.32, M S E=237.68$, 
$\left.p=.005, \eta^{2}=.063\right)$ was not dependent on whether they had a comobid anxiety disorder, MDD by Anxiety Disorder interaction: $F(1,124)=0.12$, $M S E=237.68, p=.73, \eta^{2}<.001$.

\section{Correlational analyses}

Across all participants, greater severity of current depression and anxiety symptom ratings was associated with a smaller left ear advantage for emotional recognition (depression: $r=.19, p=.04$; anxiety: $r=.20, p=.03$ ). These correlations remained significant when calculated for participants with a lifetime MDD (depression: $r=.28, p=.047$; anxiety: $r=.35, p=.01$ ), but not for those without a MDD (depression: $r=.05, p=.71$; anxiety: $r=$ $-.01, p=.93$ ). Among participants with a MDD, greater severity of depression and anxiety symptoms was significantly associated with a smaller left ear advantage for sad items (depression: $r=.30, p=.03$; anxiety: $r=.35, p=.01$ ), and poorer left ear accuracy for sad items (depression: $r=-.42, p=.002$; anxiety: $r=-.40, p=.003$ ), but not right ear accuracy (both $r=-.11, p=.42$ ). To further examine whether greater severity of current depressive symptoms in individuals with a lifetime MDD could account for their reduced left ear advantage for emotional recognition, we included only individuals with little or no depressive symptoms (i.e., combined depression $z$-scores $\leq 0$ ). The difference in PA between those with versus without a lifetime MDD was no longer significant, $t(85)=.65, p=.52$, which suggests that current depressive symptoms contributed to the reduced left ear advantage in individuals with a MDD. Age of participants was not significantly associated with PA in the emotional recognition test $(r=-.14, p=.12)$. When age was included as a covariate, the difference in PA between participants with versus without a lifetime MDD remained significant, $F(1,123)=9.43, M S E=$ 67.34, $p=.003, \eta^{2}=.071$.

\section{Consonant-vowel test}

\section{Lifetime MDD}

The expected difference in PA for syllables was found for the non-forced, forced-right and forced-left attentional conditions, Ear by Condition interaction: $F(2,236)=125.27, M S E=368.56, p<.001, \eta^{2}=.508$. Across all groups (Figure 3), there was a right ear advantage for consonant-vowels in the non-forced condition with significantly greater accuracy for right ear $(M=$ 49.6, $S D=12.0)$ than left ear $(M=34.3, S D=10.6), t(125)=8.86, p<.001$. A larger right ear advantage was found for the forced-right condition with right ear accuracy $(M=60.4, S D=17.2)$ being markedly greater than left ear accuracy $(M=23.2, S D=13.4), t(125)=14.7, p<.001)$. The PA shifted to a strong left ear advantage in the forced-left condition with left ear accuracy $(M=52.0, S D=18.6)$ being greater than right ear accuracy $(M=26.1, S D=$ 


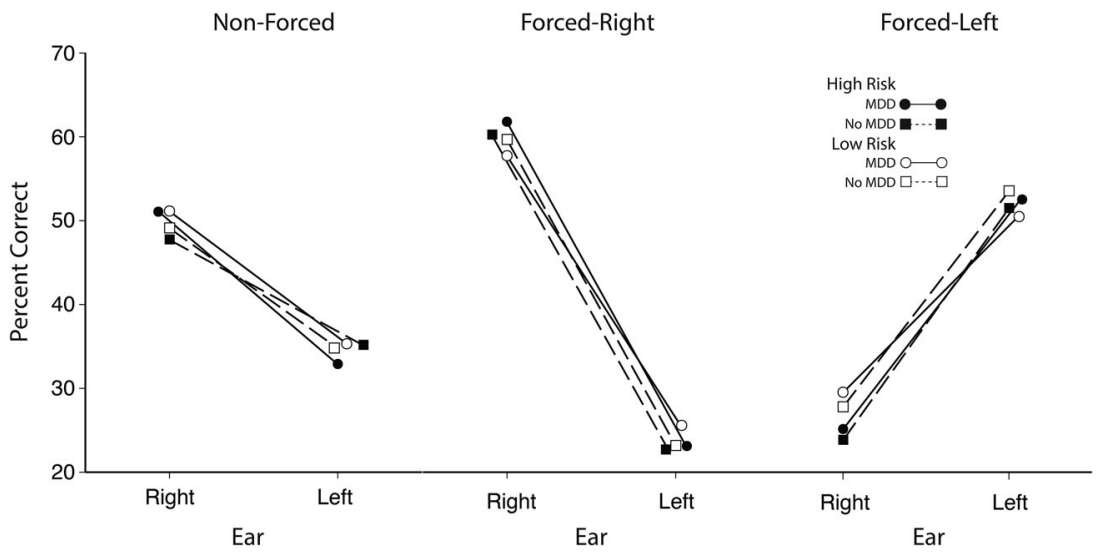

Figure 3. Mean accuracy in consonant-vowel test for the right and left ears in high-risk and low-risk groups with or without a MDD for non-forced, forced-right and forced-left conditions.

14.6), $t(125)=9.89, p<.001$. As can be seen in Figure 3 , the PA for these conditions did not differ between the high- and low-risk groups or between the participants with or without a MDD, that is, there were no significant interactions of Risk or MDD with Ear and Condition (all $p$ values $\geq .14$ ). Nor were there significant differences in overall accuracy levels between the highand low-risk groups or between participants with or without a MDD (all $p$ values $\geq .48$ ).

\section{Lifetime anxiety disorder}

There was, however, a difference in PA for syllables between participants with an anxiety disorder and those without an anxiety disorder. When lifetime Anxiety Disorder was used as a grouping variable in the ANOVA, there was a significant Anxiety Disorder by Ear interaction, $F(1,118)=5.17, M S E=284.55$, $p=.025, \eta^{2}=.042$. The right ear advantage in individuals with an anxiety disorder $(M=6.6, S D=13.2)$ was smaller compared to those with no anxiety disorder $(M=11.4, S D=14.3), t(126)=2.00, p=.049$ (Cohen's $d=.35)$. The difference in PA between those with versus without an anxiety disorder was not dependent on the attentional condition (i.e., there was no Anxiety Disorder by Ear by Condition interaction, $F(2,236)=.12, M S E=379.60, p=.88, \eta^{2}=.001$. Figure 4 shows the right and left ear accuracy levels for each group in the syllable test (averaged across condition). The smaller right ear advantage in those with an anxiety disorder was not dependent on risk status, which is indicated by lack of a significant interaction of Anxiety Disorder by Risk by Ear interaction, $F$ $(1,118)=.47, M S E=284.55, p=.49, \eta^{2}=.004$. There were also no significant effects of Generation, and a separate analysis including sex of participants as a variable did not find significant effects of this variable on the right ear 


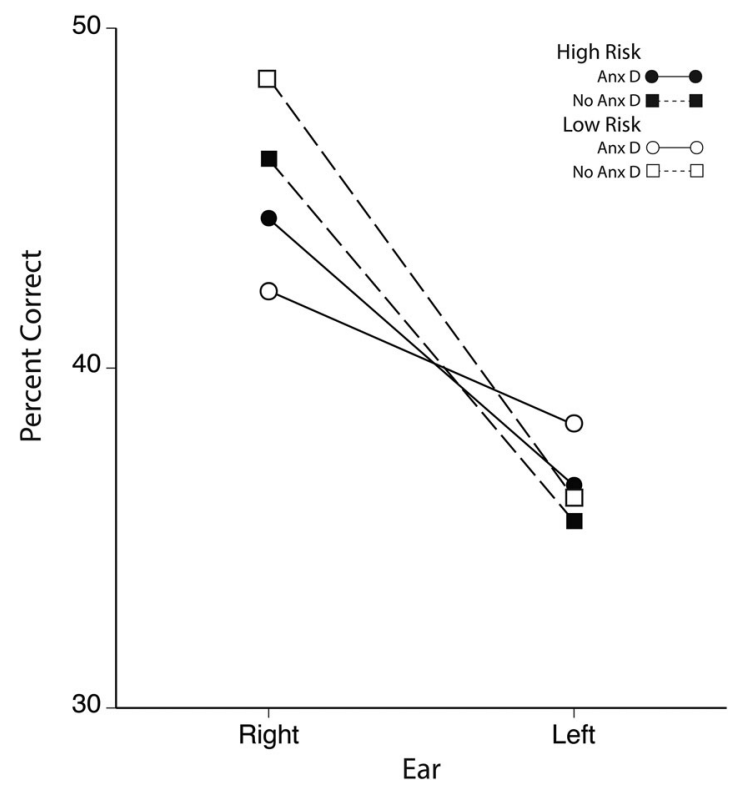

Figure 4. Mean accuracy in the consonant-vowel test (averaged over condition) for the right and left ears in high-risk and low-risk groups with or without an anxiety disorder.

advantage or accuracy levels for syllables. A further analysis found that the smaller right ear advantage in those with an anxiety disorder was not dependent on whether they had a co-morbid MDD. A $2 \times 2$ ANOVA found the same difference in the right ear advantage between those with versus without an anxiety disorder, $F(1,122)=3.62, M S E=186.15, p=.060, \eta^{2}=.029$, but no difference between those with or without a MDD, $F(1,122)=.89$, MSE $=186.15, p=.36, \eta^{2}=.007$, and no anxiety disorder by MDD interaction, $F(1$, 122) $=1.88$, MSE $=186.15, p=.17, \eta^{2}=.015$.

As can be seen in Figure 4, the reduced right ear advantage in those with an anxiety disorder was primarily due to their poorer right ear accuracy $(M=$ $43.7, S D=9.0)$ compared to those with no anxiety disorder $(M=47.2, S D=8.8)$, $t(124)=2.23, p=.028$; Cohen's $d=.40$. There was no significant difference in the left ear accuracy between those with an anxiety disorder $(M=37.1, S D$ $=8.4)$ and those with no anxiety disorder $(M=35.8, S D=8.4), t(124)=.88, p$ $=.38$. We also found a trend for an Anxiety Disorder by Condition interaction, $F(2,236)=2.98, M S E=50.76, p=.053, \eta^{2}=.025$. This interaction reflects the poorer accuracy in the forced-right condition in participants with an anxiety disorder $(M=40.7, S D=7.0)$ than in those with no anxiety disorder $(M=$ 43.0, $S D=4.4), t(124)=2.14, p=.034$, Cohen's $d=.40$, whereas there was no significant difference between these groups in accuracy for the non-forced or forced-left conditions ( $p$-values $\geq .23$ ). 


\section{Correlational analyses}

There was no significant correlation between PA for syllables (averaged across condition) and severity of current depressive $(r=-.02, p=.80)$ or anxiety $(r=.08, p=.36)$ symptom ratings. Nor did accuracy levels correlate with severity of depressive symptoms $(r=.17, p=.06)$ or anxiety symptoms $(r=.06, p=.49)$. Age was also not significantly correlated with PA or accuracy in the syllable test. To further examine whether the reduced PA for syllables in individuals having a lifetime anxiety disorder was related to greater severity of current anxiety, we included only individuals who had little or no anxiety symptoms (i.e., combined anxiety $z$-scores $\leq 0)$. Those with an anxiety disorder $(M=4.5, S D$ $=13.7$ ) continued to show a smaller right ear advantage for syllables compared to those without an anxiety disorder $(M=12.6, S D=14.4 ; t[82]=2.61, p=.01$, Cohen's $d=.58$ ). This suggests that current anxiety symptoms did not contribute to the reduced right ear advantage for individuals with an anxiety disorder.

\section{Correlations across tests}

Correlations were calculated between the PA for the emotional recognition test and the CV syllable test for the non-forced condition, where participants attend to both ears. Across all participants, there was no significant correlation between the PA for these tests $(r=.13, p=.15)$. This was also the case when correlations were calculated separately for participants with a lifetime MDD $(r=.12, p=.39)$ and those without a MDD $(r=.11, p=.37)$ or for participants with a lifetime anxiety disorder $(r=.15, p=.22)$ and those without an anxiety disorder $(r=.16, p=.22)$.

\section{Discussion}

The present study used a longitudinal data set to examine the relation of risk for depression, as well as lifetime diagnosis of MDD or anxiety disorder, on PA in emotional and verbal dichotic listening tests. For emotion testing, we used a task originally developed in Bryden's laboratory (Bryden \& MacRae, 1989), thereby demonstrating his continuing influence on current research. The verbal dichotic test, developed by Hugdahl and his associates (Hugdahl \& Andersson, 1986; Hugdahl et al., 2003), provides measures of PA for consonant-vowel syllables under different attentional conditions. We predicted that individuals at high risk for depression and those having a lifetime history of MDD would be particularly likely to show reduced PA in the emotional recognition test, whereas individuals having an anxiety disorder would be most likely to show reduced PA in the dichotic syllable test.

\section{Emotional recognition test}

We replicated prior findings of a left ear advantage for the dichotic emotional recognition test, which reflects right hemisphere dominance for prosodic 
perception (Bryden \& MacRae, 1989; Voyer et al., 2009). The overall magnitude of the left ear advantage in our multigenerational study of risk for depression was the about the same as previously found for adults (Voyer et al., 2009), and it was not related to either age or sex of participants. As predicted, individuals with a lifetime diagnosis of MDD showed less left ear advantage when compared to those with no history of a MDD. This is the first evidence that right hemisphere dominance for prosody is reduced in individuals with a lifetime MDD. Although there was no difference in the accuracy of emotional recognition between those with versus without a MDD, greater severity of current depressive symptoms was associated with poorer left ear accuracy. Given the contralateral projections from ear to hemisphere, our findings support other evidence that depression is associated with right hemisphere dysfunction for emotional processing (Bruder et al., 2002; David \& Cutting, 1990; Deldin et al., 2000; Jaeger et al., 1987; Kayser et al., 2000; Kucharska-Pietura \& David, 2003; Moratti et al., 2008). The reduced right hemisphere advantage for emotional prosody in MDD could have important implications for how depressed individuals perceive, process and respond to emotional stimuli. They may be less able to deal effectively with emotional information in real life situations.

Although the left ear advantage for emotional recognition in individuals without a lifetime MDD was present for angry, sad and happy emotions, it was largest for the negative emotions and not present at all for neutral items. This is consistent with the early findings of Bryden and MacRae (1989) for a dichotic monitoring test. There is considerable support for right hemisphere involvement in emotional prosody regardless of emotional valence (Borod, et al., 1998), but there is also evidence of emotion-specific differences in hemispheric lateralization (for a review, see Gadea et al., 2011). Most notably, the valence hypothesis postulates that right frontal activation is associated with the experience of negative or withdrawal emotions such as sadness and fear, whereas left frontal activation is associated with approach emotions such as happiness and anger. The findings of Grimshaw, Séguin, and Godfrey (2009) for a verbal dichotic listening task in which words were spoken with different emotional intonation were most consistent with this valence hypothesis. They found that the right ear advantage for words with neutral emotional tone was attenuated when words were spoken with sad prosody, but not for angry or happy prosody. In the present study, the left ear advantage in the emotional recognition test was reduced in MDD, but this was only significant for words with sad prosody. One hypothesis is that sad items activate a right frontotemporal network associated with this negative emotional experience, but right hemisphere dysfunction in MDD reduces the PA for sad prosody. Another possible explanation is that depressed individuals attempt to divert their attention from sad stimuli, thereby reducing the attentional bias to the left ear that would be hypothesized on the basis of Kinsbourne's (1970) model. 
Contrary to our prediction, we did not did find a difference in the left ear advantage or accuracy of emotional recognition between individuals at high risk versus low risk for developing a depressive disorder. This prediction was based on resting EEG and structural MRI evidence of right hemisphere deficits in individuals at risk for MDD (Bruder et al., 2012; Peterson et al., 2009). These EEG and MRI data were, however, acquired in prior waves of this longitudinal study, with EEG tests ending in 2002 and MRI tests ending in 2007, and no behavioural laterality data for emotional processing were obtained in these waves. The dichotic listening tests were obtained in the latest wave ending in 2015 and indicate that reduced right hemisphere processing of emotional prosody is present in individuals with lifetime history of MDD, regardless of their risk for MDD. The reduced PA for emotional recognition was also correlated with severity of current depressive symptoms. This suggests that it is more state dependent than the abnormalities seen for resting EEG or MRI, which were not dependent on having a MDD. There are a number of possible explanations for the difference in the findings for $E E G, M R I$ and dichotic listening. First, the EEG measures were acquired in a resting state and not during emotional processing, and the MRI measures were for brain structure but not function. The PA findings may reflect reactivity to the dichotic emotional stimuli, which would not be seen for the EEG or MRI measures. Second, the dichotic listening tests were obtained in the latest wave of the study about 30 years after baseline assessment of the probands. Many of the offspring and grandchildren will have now passed through the age of risk for developing a MDD, which may make it more likely to find abnormalities in those with lifetime MDD. Third, the emotional recognition test may not be sensitive enough to detect differences between those at high versus low risk for depression. In absolute terms, the left ear advantage for emotional recognition is small compared to the right ear advantage for syllables (see also Voyer, 2011 on this point). Differences between high- and lowrisk individuals might be detected with more emotionally arousing stimuli, or when using more direct electrophysiological or fMRI measures of hemispheric asymmetry. This is now being investigated in the context of this multigenerational high-risk study by measuring ERPs to emotionally arousing negative stimuli that yield enhanced late positive brain potentials, particularly over right hemisphere regions in healthy adults, but which are reduced in depressed patients (Kayser et al., 2000).

Individuals having a lifetime diagnosis of an anxiety disorder did not differ from those without an anxiety disorder in either left ear advantage or accuracy on the emotional recognition test. This suggests that reduced right hemisphere processing of emotional stimuli is specific to those having a depressive disorder. In a prior study (Bruder et al., 2004), patients with social phobia also did not differ from those without social phobia in right hemisphere advantage for non-verbal dichotic listening. Although having a lifetime anxiety disorder 
did not affect performance, greater severity of current anxiety symptoms was associated with less left ear advantage. This may reflect the lack of specificity of anxiety and depression symptom ratings on standard scales, which are significantly correlated. There is, however, evidence that depressed patients having an anxiety disorder differ from those without this comorbidity in their hemispheric asymmetry for dichotic listening (Bruder et al., 1999) and resting EEG alpha asymmetry (Bruder et al., 1997; Kentgen et al., 2000). To examine the potential influence of comorbidity, we performed an analysis grouping patients on the basis of both lifetime diagnoses of MDD and anxiety disorder. The reduced left ear advantage for sad emotional prosody was found for MDD with or without a comorbid anxiety disorder.

\section{Consonant-vowel test}

We found the expected strong right ear advantage in the non-forced and forced-right consonant-vowel conditions, which shifted to a left ear advantage when attention was directed to the left side. This replicates the original findings of Hugdahl et al. (2003) for healthy adults. There was no difference in the PA for these conditions between individuals at high or low risk for depression or between those with versus without a lifetime diagnosis of MDD. In contrast, individuals with a lifetime diagnosis of an anxiety disorder had a smaller right ear advantage for syllables compared to those without an anxiety disorder. Although the effect size was in the small to moderate range, this group difference is consistent with findings for patients having an anxiety disorder with or without a depressive disorder (Bruder et al., 1999, 2004). The reduced PA was due to the significantly poorer accuracy for right ear, but not left ear, syllables in those with an anxiety disorder. Moreover, they had poorer accuracy in the forced-right condition, but not in the non-forced or forced-left conditions. Although these findings suggest that lifetime anxiety disorder is associated with a deficit in contralateral left hemisphere processing of verbal stimuli, other explanations are possible. Asbjörnsen et al. (1992) found that threat of electric shock abolished the right ear advantage for syllables in healthy adults. They argue that the emotional arousal effect of threat of shock differentially activates the right hemisphere, and also facilitates callosal transfer of left ear signal, which should increase left ear correct reports. They found that the reduction of right ear advantage was due to both an increase in left ear correct and a decrease in right ear correct reports, which supports their interpretation. However, we did not find a difference in left ear accuracy in individuals with a lifetime anxiety disorder and they also did not differ from those with no anxiety disorder in right hemisphere advantage for emotional processing. Our findings are more supportive of the hypothesis that reduced right ear advantage in anxiety disorders is associated with dysfunction of left 
hemisphere regions mediating verbal processing. This is likely related to processes in the temporal-parietal region, where EEG alpha asymmetry has been linked to the right ear advantage for dichotic syllables or words (Bruder et al., 2001; Davidson \& Hugdahl, 1996).

The different conditions in the consonant-vowel syllable test also provided information concerning the influence of attention on dichotic listening performance (Hugdahl et al., 2003). Individuals at high risk for depression and those having a lifetime MDD did not show abnormal attentional modulation of performance in the syllable test. Although the reduction of right ear advantage in those with a lifetime anxiety disorder did not depend on attention, accuracy of performance was significantly poorer in those with an anxiety disorder compared to those without an anxiety disorder in the forced-right condition, but not the non-forced and forced-left conditions. This suggests that left hemisphere dysfunction in individuals with an anxiety disorder may impair the biasing of attention to the right ear during verbal processing.

\section{Conclusions}

We found evidence of a double dissociation of brain laterality deficits between individuals having a lifetime diagnosis of a MDD and those having a lifetime anxiety disorder. MDD was associated with reduced right hemisphere advantage for emotional prosody but no difference in left hemisphere advantage for consonant-vowel syllables. In contrast, having an anxiety disorder was associated with reduced left hemisphere advantage for syllables but no difference in right hemisphere advantage for emotional prosody. These abnormalities of brain laterality were present even among those who did not have the risk conveyed by a depressed parent or grandparent, and therefore do not appear to be risk factors for developing these disorders. The reduced right hemisphere advantage in individuals with lifetime MDD was related to current depressive symptoms, being most evident in those with greater severity of depression. In contrast, the reduced left hemisphere advantage in individuals with lifetime anxiety disorder was not related to severity of current anxiety symptoms, and was present among those with little or no anxiety symptoms, which suggests that it persists despite the resolution of their psychopathology.

These findings together indicate that lifetime diagnosis of MDD and anxiety disorders have a differential impact on lateralized hemispheric processing of verbal and emotional information. Although we did not find evidence for effects of comorbidity of these disorders on brain laterality, additional studies with larger samples are needed to further examine this in separate groups having a MDD but no anxiety disorder, an anxiety disorder but no MDD, or comorbidity of a MDD and anxiety disorder. The Research Domain 
Criteria (RDoC) project aims to develop new ways of classifying mental disorders on the basis of dimensions of behavioural and neurobiological measures (Cuthbert, 2015). Measures of brain laterality for processing emotional prosody with negative valence or processing language-related information could contribute to this effort. In this context, it may be of value to examine what behavioural or neurobiological measures cut across depressive and anxiety disorders or are specific to these disorders. Our findings suggest some degree of specificity for brain laterality deficits in depressive and anxiety disorders.

Lastly, Bryden (1990) raised the issue of whether the apparent complementarity between hemispheric specialization for verbal and non-verbal processing stems from related asymmetric processes, that is reflect causal complementarity, or whether these processes are unrelated or only reflect statistical complementarity. The causal complementarity model predicts a negative correlation between the verbal and non-verbal laterality, where individuals who show a left hemisphere advantage for verbal material would show right hemisphere advantage for non-verbal material. The lack of significant correlation between PA for dichotic syllable and emotional recognition tests in present study provides no support for causal complementarity, which agrees with most prior studies (Bryden, 1990). However, the verbal and non-verbal tasks in these studies used different processing requirements. In contrast, Voyer, Bowes, and Techentin (2008) using more comparable tasks, yielding a left ear advantage for sarcastic statements and a right ear advantage for sincere statements, found a strong negative correlation supporting causal complementarity. Regardless of above considerations, the present study shows that dichotic listening methods developed by Bryden can provide new insights into deficits in lateralized hemispheric processing of emotional information in individuals having a depressive disorder, 20 years after his passing.

\section{Acknowledgements}

The authors thank Kenneth Hugdahl of the University of Bergen, Norway, for providing a $C D$ and instructions for administering the dichotic syllable test and for his comments on this manuscript.

\section{Disclosure statement}

No potential conflict of interest was reported by the authors.

\section{Funding}

This research was funded by NIMH Grant MH036197 (MMW). 


\section{References}

Asbjörnsen, A., Hugdahl, K., \& Bryden, M. P. (1992). Manipulations of subjects' level of arousal in dichotic listening. Brain and Cognition, 19, 183-194. doi:10.1016/02782626(92)90044-M

Borod, J., Cicero, B. A., Obler, L. K., Welkowitz, J., Erhan, H. M., Santschi, C., ... Whalen, J. R. (1998). Right hemisphere emotional perception: evidence across multiple channels. Neuropsychology, 12(3), 446-458.

Bruder, G. E. (2003). Frontal and parietotemporal asymmetries in depressive disorders: Behavioral, electrophysiologic and neuroimaging findings. In K. Hugdahl \& R. J. Davidson (Eds.), The asymmetrical brain (pp. 719-742). Cambridge, MA: MIT Press.

Bruder, G. E., Bansal, R., Tenke, C. E., Liu, J., Hao, X., Warner, V., ... Weissman, M. M. (2012). Relationship of resting EEG with anatomical MRI measures in individuals at high and low risk for depression. Human Brain Mapping, 33(6), 1325-1333. doi:10. 1002/hbm.21284

Bruder, G. E., Fong, R., Tenke, C. E., Leite, P., Towey, J. P., Stewart, J. W., ... Quitkin, F. M. (1997). Regional brain asymmetries in major depression with or without an anxiety disorder: A quantitative electroencephalographic study. Biological Psychiatry, 41(9), 939-948. doi:10.1016/S0006-3223(96)00260-0

Bruder, G. E., Quitkin, F. M., Stewart, J. W., Martin, C., Voglmaier, M. M., \& Harrison, W. M. (1989). Cerebral laterality and depression: Differences in perceptual asymmetry among diagnostic subtypes. Journal of Abnormal Psychology, 98, 177-186. doi:10. 1037/0021-843X.98.2.177

Bruder, G. E., Schneier, F. R., Stewart, J. W., McGrath, P. J., \& Quitkin, F. (2004). Left hemisphere dysfunction during verbal dichotic listening tests in patients who have social phobia with or without comorbid depressive disorder. American Journal of Psychiatry, 161, 72-78. doi:10.1176/appi.ajp.161.1.72

Bruder, G. E., Stewart, J. W., McGrath, P. J., Ma, G. J., Wexler, B. E., \& Quitkin, F. M. (2002). Atypical depression: Enhanced right hemispheric dominance for perceiving emotional chimeric faces. Journal of Abnormal Psychology, 111(3), 446-454. doi:10. 1037/0021-843X.111.3.446

Bruder, G. E., Stewart, J. W., Tenke, C. E., McGrath, P. J., Leite, P., Bhattacharya, N., \& Quitkin, F. M. (2001). Electroencephalographic and perceptual asymmetry differences between responders and nonresponders to an SSRI antidepressant. Biological Psychiatry, 49, 416-425. doi:10.1016/S0006-3223(00)01016-7

Bruder, G. E., Tenke, C. E., Stewart, J. W., Towey, J. P., Leite, P., Voglmaier, M., \& Quitkin, F. M. (1995). Brain event-related potentials to complex tones in depressed patients: Relations to perceptual asymmetry and clinical features. Psychophysiology, 32(4), 373-381. doi:10.1111/j.1469-8986.1995.tb01220.x

Bruder, G. E., Tenke, C. E., Warner, V., Nomura, Y., Grillon, C., Hille, J., ... Weissman, M. M. (2005). Electroencephalographic measures of regional hemispheric activity in offspring at risk for depressive disorders. Biological Psychiatry, 57(4), 328-335. doi:10. 1016/j.biopsych.2004.11.015

Bruder, G. E., Tenke, C. E., Warner, V., \& Weissman, M. M. (2007). Grandchildren at high and low risk for depression differ in EEG measures of regional brain asymmetry. Biological Psychiatry, 62(11), 1317-1323. doi:10.1016/j.biopsych.2006.12.006

Bruder, G. E., Wexler, B. E., Stewart, J. W., Price, L. H., \& Quitkin, F. M. (1999). Perceptual asymmetry differences between major depression with or without a comorbid anxiety disorder: A dichotic listening study. Journal of Abnormal Psychology, 108 (2), 233-239. doi:10.1037/0021-843X.108.2.233 
Bryden, M. P. (1982). Laterality: Functional asymmetry in the intact brain. New York, NY: Academic Press.

Bryden, M. P. (1990). Choosing sides: The left and right of the normal brain. Canadian Psychology, 31, 297-309.

Bryden, M. P., \& MacRae, L. (1989). Dichotic laterality effects obtained with emotional words. Neuropsychiatry, Neuropsychology and Behavioral Neurology, 1, 171-176.

Cuthbert, B. N. (2015). Research domain criteria: Toward future psychiatric nosologies. Dialogues in Clinical Neuroscience, 17, 89-97.

David, A. S., \& Cutting, J. C. (1990). Affect, affective disorder and schizophrenia. A neuropsychological investigation of right hemisphere function. British Journal of Psychiatry, 156, 491-495. doi:10.1192/bjp.156.4.491

Davidson, R. J., \& Hugdahl, K. (1996). Baseline asymmetries in brain electrical activity predict dichotic listening performance. Neuropsychology, 10, 241-246. doi:10.1037/ 0894-4105.10.2.241

Deldin, P. J., Keller, J., Gergen, J. A., \& Miller, G. A. (2000). Right-posterior face processing anomaly in depression. Journal of Abnormal Psychology, 109, 116-121. doi:10.1037/ 0021-843X.109.1.116

Gadea, M., Espert, R., Salvador, A., \& Martí-Bonmatí, L. (2011). The sad, the angry, and the asymmetrical brain: Dichotic listening studies of negative affect and depression. Brain and Cognition, 76, 294-299. doi:10.1016/j.bandc.2011.03.003

Grimshaw, G. M., Séguin, J. A., Godfrey, H. K. (2009). Once more with feeling: The effects of emotional prosody on hemispheric specialization for linguistic processing. Journal of Neurolinguistics, 22, 313-326. doi:10.1016/j.jneuroling.2008.10.005

Hamilton, M. (1959). The assessment of anxiety states by rating. British Journal of Medical Psychology, 32, 50-55.

Hamilton, M. (1967). Development of a rating scale for primary depressive illness. British Journal of Social and Clinical Psychology, 6, 278-296.

Heller, W., Etienne, M. A., \& Miller, G. A. (1995). Patterns of perceptual asymmetry in depression and anxiety: Implications for neuro-psychological models of emotion and psychopathology. Journal of Abnormal Psychology, 104, 327-333. doi:10.1037/ 0021-843X.104.2.327

Heller, W., \& Nitschke, J. B. (1998). The puzzle of regional brain activity in depression and anxiety: The importance of subtypes and comorbidity. Cognition and Emotion, 12, 421-447. doi:10.1080/026999398379664

Henriques, J. B., \& Davidson, R. J. (1990). Regional brain electrical asymmetries discriminate between previously depressed and healthy control subjects. Journal of Abnormal Psychology, 99, 22-31. doi:10.1037/0021-843X.99.1.22

Henriques, J. B., \& Davidson, R. J. (1991). Left frontal hypoactivation in depression. Journal of Abnormal Psychology, 100, 535-545. doi:10.1037/0021-843X. 100.4.535

Hugdahl, K., \& Andersson, L. (1986). The "forced-attention paradigm" in dichotic listening to CV-syllables: A comparison between adults and children. Cortex, 22, 417-432. doi:10.1016/S0010-9452(86)80005-3

Hugdahl, K., Rund, B. R., Lund, A., Asbjørnsen, A., Egeland, J., Landrø, N. I., ... Sundet, K. (2003). Attentional and executive dysfunctions in schizophrenia and depression: Evidence from dichotic listening performance. Biological Psychiatry, 53, 609-616. doi:10.1016/S0006-3223(02)01598-6

Jaeger, J., Borod, J. C., \& Peselow, E. (1987). Depressed patients have atypical biases in the perception of emotional chimeric faces. Journal of Abnormal Pyschology, 96, 321-324. doi:10.1037/0021-843X.96.4.321 
Kaufman, J., Birmaher, B., Brent, D., Rao, U., Flynn, C., Moreci, P., ... Ryan, N. (1997). The schedule for affective disorders and schizophrenia for school-age children-Present and lifetime version (K-SADS-PL): Initial reliability and validity data. Journal of the American Academy of Child and Adolescent Psychiatry, 36, 980-988.

Kayser, J., Bruder, G. E., Tenke, C. E., Stewart, J. W., \& Quitkin, F. M. (2000). Event related potentials (ERPs) to hemifield presentations of emotional stimuli: Differences between depressed patients and healthy adults in P3 amplitude and asymmetry. International Journal of Psychophysiology, 36, 211-236. doi:10.1016/S0167-8760(00) 00078-7

Kentgen, L. M., Tenke, C., Pine, D. S., Fong, R., Klein, R. G., Bruder, G. E. (2000). Electroencephalographic asymmetries in adolescents with major depression: Influence of comorbidity with anxiety disorders. Journal of Abnormal Psychology, 109(4), 797-802. doi:10.1037/0021-843X.109.4.797

Kinsbourne, M. (1970). The cerebral basis of lateral asymmetries in attention. Acta Psychologia, 33, 193-201. doi:10.1016/0001-6918(70)90132-0

Kucharska-Pietura, K., \& David, A. S. (2003). The perception of emotional chimeric faces in patients with depression, mania and unilateral brain damage. Pyschological Medicine, 33, 739-745. doi:10.1017/S0033291702007316

Leckman, J. F., Sholomskas, D., Thompson, D., Belanger, A., \& Weissman, M. M. (1982). Best estimate of lifetime diagnosis: A methodological study. Archives of General Psychiatry, 39, 879-883. doi:10.1001/archpsyc.1982.04290080001001

Levy, J., Heller, W., Banich, M., \& Burton, L. A. (1983). Are variations among right handed individuals in perceptual asymmetries caused by characteristic arousal differences between hemispheres? Journal of Experimental Psychology: Human Perception and Performance, 9, 329-359. doi:10.1037/0096-1523.9.3.329

Liotti, M., Sava, D., Rizzolatti, G., \& Caffarra, P. (1991). Differential hemispheric asymmetries in depression and anxiety: A reaction-time study. Biological Psychiatry, 29, 887899. doi:10.1016/0006-3223(91)90055-Q

Mannuzza, S., Fyer, A. J., Klein, D. F., \& Endicott, J. (1986). Schedule for affective disorders and schizophrenia-lifetime version modified for the study of anxiety disorders (SADS-LA): Rational and conceptual development. Journal of Psychiatric Research, 20, 317-325. doi:10.1016/0022-3956(86)90034-8

Moratti, S., Rubio, G., Campo, P., Keil, A., Ortiz, T. (2008). Hypofunction of right temporoparietal cortex during emotional arousal in depression. Archives of General Psychiatry, 65, 532-541. doi:10.1001/archpsyc.65.5.532

Moscovitch, M., Strauss, E., \& Olds, J. (1981). Handedness and dichotic listening performance in patients with unipolar endogenous depression who received ECT. American Journal of Psychiatry, 138, 988-990. doi:10.1176/ajp.138.7.988

Oldfield, R. C. (1971). The assessment and analysis of handedness: The Edinburgh inventory. Neuropsychologia, 9, 97-113. doi:10.1016/0028-3932(71)90067-4

Orvaschel, H., Puig-Antich, J., Chambers, W., Tabriz, M. A., \& Johnson, R. (1982). Retrospective assessment of prepubertal major depression with the Kiddie-SADSE. Journal of the American Academy of Child and Adolescent Psychiatry, 21, 392397. doi:10.1016/S0002-7138(09)60944-4

Perrin, S., \& Last, C. G. (1992). Do childhood anxiety measures measure anxiety? Journal of Abnormal Child Psychology, 20, 567-578.

Peterson, B. S., Warner, V., Bansal, R., Zhu, H., Hao, X., Liu, J., ... Weissman, M. M. (2009). Cortical thinning in persons at increased familial risk for major depression. Proceedings of the National Academy of Sciences of the United States of America, 106, 6273-6278. doi:10.1073/pnas.0805311106 
Pine, D., Kentgen, L. M., Bruder, G. E., Leite, P., Bearman, K., Ma, Y., \& Klein, R. G. (2000). Cerebral laterality in adolescent major depression. Psychiatry Research, 93, 135-144. doi:10.1016/S0165-1781(00)00101-3

Poznanski, E. O., Freeman, L. N., Mokros, H. B. (1985). Children's depression rating scale revised. Psychopharmacology Bulletin, 21, 979-989.

Reid, S. A., Duke, L. M., \& Allen, J. B. (1998). Resting frontal electroencephalographic asymmetry in depression: Inconsistencies suggest the need to identify mediating factors. Psychophysiology, 35, 389-404. doi:10.1111/1469-8986.3540389

Schaffer, C. E., Davidson, R. J., Saron, C. (1983). Frontal and parietal electroencephalogram asymmetry in depressed and nondepressed subjects. Biological Psychiatry, $18,753-762$.

Shankman, S. A., Tenke, C. E., Bruder, G. E., Durbin, C. E., Hayden, E. P., \& Klein, D. N. (2005). Low positive emotionality in young children: Association with EEG asymmetry. Developmental Psychopathology, 17(1), 85-98. doi:10.1017/S0954579405050054

Voyer, D. (2003). Reliability and magnitude of perceptual asymmetries in a dichotic word recognition task. Neuropsychology, 17, 393-401. doi:10.1037/0894-4105.17.3.39

Voyer, D. (2011). Sex differences in dichotic listening. Brain and Cognition, 76, 245-255. doi:10.1016/j.bandc.2011.02.001

Voyer, D., Bowes, A., \& Soraggi, M. (2009). Response procedure and laterality effects in emotion recognition: Implications for models of dichotic listening. Neuropsychologia, 47, 23-29. doi:10.1016/j.neuropsychologia.2008.08.022

Voyer, D., Bowes, A., Techentin, C. (2008). On the perception of sarcasm in dichotic listening. Neuropsychology, 22, 390-399. doi:10.1037/0894-4105.22.3.390

Voyer, D., Russell, A., \& McKenna, J. (2002). On the reliability of laterality effects in a dichotic emotion recognition task. Journal of Clinical and Experimental Neuropsychology, 24, 605-614. doi:10.1076/jcen.24.5.605.1007

Wale, J., \& Carr, V. (1990). Differences in dichotic listening asymmetries in depression according to symptomatology. Journal of Affective Disorders, 18, 1-9. doi:10.1016/ 0165-0327(90)90111-K

Warner, V., Mufson, L., \& Weissman, M. M. (1995). Offspring at high and low risk for depression and anxiety: Mechanisms of psychiatric disorder. Journal of the American Academy of Child and Adolescent Psychiatry, 34, 786-797. doi:10.1097/ 00004583-199506000-00020

Warner, V., Weissman, M. M., Mufson, L., \& Wickramaratne, P. J. (1999). Grandparents, parents, and grandchildren at high risk for depression: A three-generation study. Journal of the American Academy of Child and Adolescent Psychiatry, 38, 289-296. doi:10.1097/00004583-199903000-00016

Weissman, M. M., Warner, V., Wickramaratne, P., Moreau, D., \& Olfson, M. (1997). Offspring of depressed parents: 10 years later. Archives of General Psychiatry, 54, 932-940. doi:10.1001/archpsyc.1997.01830220054009

Weissman, M. M., Wickramaratne, P., Nomura, P., Warner, V., Pilowsky, D., \& Verdeli, H. (2006). Offspring of depressed parents: 20 years later. American Journal of Psychiatry, 163, 1001-1008. doi:10.1176/ajp.zool.163.6.1001

Weissman, M. M., Wickramaratne, P., Nomura, P., Warner, V., Verdeli, H., Pilowsky, D. J., ... Bruder, G. E. (2005). Families at high and low risk for depression: A 3-generation study. Archives of General Psychiatry, 62, 29-36. doi:10.1001/archpsyc.62.1.29 